\title{
A GENERALIZATION OF LE POTIER'S VANISHING THEOREM
}

\author{
F. LAYTIMI AND W. NAHM
}

\section{IntRoduction}

Consider a vector bundle $E$ of rank $d$ over a compact complex manifold manifold $X$ of dimension $n$, and a partition $R=\left(r_{1}, r_{2}, \ldots, r_{m}\right)$ of weight $r=\sum_{i=1}^{m} r_{i}=|R|$, where the $r_{i}$ are strictly positive integers with $r_{i} \geq r_{i+1}$ and $m \leq d$. We call $m$ the length of $R$.

Let $\wedge_{R}=\mathcal{S}_{\tilde{R}}$ be the Schur functor (for the definition see [M, p.45]) corresponding to the transpose $\tilde{R}$ of $R$. Schur functors were initially defined on the category of vector spaces and linear maps, but by functoriality the definition carries over to vector bundles on $X$.

We prove the following vanishing theorems for cohomology groups:

Theorem 1.1. For any partition $R=\left(r_{1}, r_{2}, \ldots, r_{m}\right)$,

$H^{p, q}\left(X, \wedge_{R} E\right)=0$ if $\wedge_{R} E$ is ample and $p+q-n>\sum_{i=1}^{m} r_{i}\left(d-r_{i}\right)$.

If $m=1$ we get Le Potier vanishing theorem [LP1](see section 2).

Corollary 1.2. $H^{p, q}\left(X, \otimes_{i=1}^{l} \mathcal{S}^{k_{i}} E \otimes_{j=1}^{m} \wedge^{s_{j}} E\right)=0$

$$
\begin{aligned}
& \text { if } \otimes_{i=1}^{l} \mathcal{S}^{k_{i}} E \otimes_{j=1}^{m} \wedge^{s_{j}} E \text { is ample and } \\
& p+q-n>\sum_{j=1}^{m} s_{j}\left(d-s_{j}\right)+(d-1) \sum_{i=1}^{l} k_{i} .
\end{aligned}
$$

In [LP2] Le Potier introduced a very useful tool for the derivation of such theorems. It is based on the Borel-Le Potier spectral sequence, a term introduced by Demailly [D1].

Given a sequence of integers $0=s_{0}<s_{1}<s_{2}<\ldots<s_{l} \leq d$, and a complex vector space $V$ of dimension $d$, let $\mathcal{F} l_{s_{1}, \ldots, s_{l}}(V)=\mathcal{F} l_{s}(V)$ be the variety of partial flags

$$
V_{s_{l}} \subset V_{s_{l-1}} \subset \ldots \subset V_{s_{1}} \subset V, \quad \operatorname{codim} V_{s_{i}}=s_{i} .
$$

1991 Mathematics Subject Classification. 14F17. 
This manifold carries canonical vector bundles $Q_{i}$ with fibers $V_{s_{i-1}} / V_{s_{i}}$. Let $Y=\mathcal{F} l_{s}(E)$ be the natural fibered variety with projection

$\pi: Y \rightarrow X$ and fibers $\mathcal{F} l_{s}\left(E_{x}\right), x \in X$.

We also denote by $Q_{i}$ the corresponding vector bundle over $Y$. For partitions $\Lambda_{i}$, a vector bundle of the form $\otimes_{i} \mathcal{S}_{\Lambda_{i}}\left(Q_{i}\right)$ over $Y$ will be called of Schur type.

The projection $\pi$ yields a filtration of the bundle $\Omega_{Y}^{P}$ of exterior differential forms of degree $P$ on $Y$, namely

$$
F^{p}\left(\Omega_{Y}^{P}\right)=\pi^{*} \Omega_{X}^{p} \wedge \Omega_{Y}^{P-p}
$$

The corresponding graded bundle is given by

$$
F^{p}\left(\Omega_{Y}^{P}\right) / F^{p+1}\left(\Omega_{Y}^{P}\right)=\pi^{*} \Omega_{X}^{p} \otimes \Omega_{Y / X}^{P-p},
$$

where $\Omega_{Y / X}^{P-p}$ is the bundle of relative differential forms of degree $P-$ $p$. For a given line bundle $\mathcal{L}$ over $Y$, the filtration on $\Omega_{Y}^{P}$ induces a filtration on $\Omega_{Y}^{P} \otimes \mathcal{L}$. This latter filtration yields the Borel-Le Potier spectral sequence, which abuts to $H^{P, q}(Y, \mathcal{L})$.

It is given by the data $X, Y, \mathcal{L}, P$ and will be denoted by ${ }^{P} \mathcal{E}_{B}$. Its $\mathcal{E}_{1}$-terms

$$
{ }^{P} \mathcal{E}_{1, B}^{p, q-p}=H^{q}\left(Y, \pi^{*}\left(\Omega_{X}^{p}\right) \otimes \Omega_{Y / X}^{P-p} \otimes \mathcal{L}\right)
$$

can be calculated as limit groups of the Leray spectral sequence ${ }^{p, P} \mathcal{E}_{L}$ associated to the projection $\pi$, for which

$$
{ }^{p, P} \mathcal{E}_{2, L}^{q-j, j}=H^{p, q-j}\left(X, R^{j} \pi_{*}\left(\Omega_{Y / X}^{P-p} \otimes \mathcal{L}\right)\right)
$$

For a suitably chosen ample line bundle $\mathcal{L}$ (see section 6) of Schur type and for $P-p=0$ one obtains

$$
{ }^{P} \mathcal{E}_{1, B}^{P, q-P}=H^{P, q}\left(X, \wedge_{R} E\right) .
$$

Moreover, under the condition

$$
\text { (*) } P+q>n+\sum_{i=1}^{m} r_{i}\left(d-r_{i}\right)
$$

the corresponding Borel-le Potier spectral sequence will be shown to degenerate at ${ }^{P} \mathcal{E}_{1, B}^{P, q-P}$, in the sense that all ${ }^{P} d_{i, B}$ mapping to and from ${ }^{P} \mathcal{E}_{i, B}^{P, q-P}$ are zero, such that $H^{P, q}\left(X, \Lambda_{R} E\right)$ is a subquotient of $H^{P, q}(Y, \mathcal{L})$. The latter group vanishes by the Kodaira-Akizuki-Nakano vanishing theorem.

The map ${ }^{P} d_{i, B}$ from ${ }^{P} \mathcal{E}_{i, B}^{P, q-P}$ is zero by construction, since $F^{p}\left(\Omega_{Y}^{P}\right)=$ 0 for $p>P$. For the maps to ${ }^{P} \mathcal{E}_{i, B}^{P, q-P}$ we shall use an induction argument to show that their sources vanish under the condition $(*)$. 
Because of the Leray spectral sequence, these sources are given by subquotients of groups of type $\oplus_{j} H^{p, q-j}\left(X, R^{j} \pi_{*}\left(\Omega_{Y / X}^{P-p} \otimes \mathcal{L}\right)\right)$.

Since $\Omega_{Y / X}^{P-p} \otimes \mathcal{L}$ is of Schur type, the vector bundle $R^{j} \pi_{*}\left(\Omega_{Y / X}^{P-p} \otimes \mathcal{L}\right)$ on $X$ is given by a Schur functor applied to $E$. This Schur functor can be calculated for the case where $X$ is a point and $E$ a vector space $V$. Thus the core of our proof will be the evaluation of the groups $H^{P-p, j}\left(\mathcal{F} l_{s}(V), \mathcal{L}\right)$. For that, we need a number of technical preparations.

In section 3 we explain some basic tools, in particular concerning Young diagrams, which label the Schur functors. In section 4 we reformulate the Littlewood-Richardson rules for the tensor product of two Schur functors. This goes slightly beyond what we need for the proof, but should have independent interest. In section 5 we consider the relevant cohomology groups on partial flag varieties, in particular on the Grassmannians. Section 6 contains the proof of the main theorem.

\section{SOME KNOWn RESULtS}

Vanishing theorems for ample vector bundles play a crucial role in algebraic geometry and its applications.

Let us recall the most important ones.

Theorem 2.1 (Kodaira - Akizuki - Nakano ). [KAN]

Let $L$ be an ample line bundle on an $n$-dimensional projective manifold $X$.

Then $H^{p, q}(X, L)=0$ for $p+q>n$.

The special case $p=n$ is due to Kodaira. In this case the ampleness condition can be relaxed to yield the Kawamata-Viehweg vanishing theorem $[\mathrm{KV}]$.

The extension of these results to vector bundles of higher rank is due essentially to Le Potier.

In the sequel, $E$ is a vector bundle of rank $d$ on a $n$-dimensional compact complex manifold $X$.

Theorem 2.2 (Le Potier). [LP2]

If $E$ is ample, then $H^{n, q}\left(X, \wedge^{r} E\right)=0$ for $q>d-r$.

Theorem 2.3 (Le Potier). [LP1]

If $\wedge^{r} E$ is ample, then $H^{p, q}\left(X, \wedge^{r} E\right)=0$ for $p+q-n>r(d-r)$.

Although Le Potier states this theorem under the hypothesis that $E$ is ample, his proof requires the weaker hypothesis that $\wedge^{r} E$ be ample. 
Indeed, in his proof he needs $\operatorname{det} Q$ on $G_{r}(E)$ to be ample but $\operatorname{det} Q=$ $\left.\mathcal{O}_{\mathbb{P}\left(\wedge^{r} E\right)}(1)\right|_{G_{r}(E)}$. Thus $\wedge^{r} E$ ample implies that $\operatorname{det} Q$ is ample.

Theorem 2.4 (Sommese). [S]

Let $E_{j}, 1 \leq j \leq m$ be ample vector bundles of rank $d_{j}$. Then

$$
H^{p, q}\left(X, \otimes_{j=1}^{m} \wedge^{s_{j}} E_{j}\right)=0 \quad \text { if } \quad p+q-n>\sum_{j=1}^{m} s_{j}\left(d_{j}-s_{j}\right) .
$$

Note that Corollary 1.2 gives Sommese's vanishing theorem with $E_{j}=E$ for all $j$, under weaker assumption.

For vectors bundles tensored with powers of $\operatorname{det} E$, one obtains less restrictive vanishing conditions. The best known case is

Theorem 2.5 (Griffiths). $[G]$

Let $E$ be ample. Then $\left.H^{n, q}\left(X, S^{r} E \otimes \operatorname{det} E\right)\right)=0$ for $q>0$.

The finite dimensional irreducible representations of $G l(V)$, where $V$ is vector space of dimension $d$ are in correspondence with partitions of length at most $d$. We denote the irreducible $G l(V)$-module corresponding to the partition $R$ by $S_{R}(V)$, and call it the Schur functor of $V$.

In particular $S^{k} V=\mathcal{S}_{(k)} V$, and $\wedge^{h} V=\mathcal{S}_{\left(\mathcal{S}^{(1,1, \ldots, 1)}\right.} V$.

An extension of the last theorem to Schur functors is

Theorem 2.6 (Demailly). [D1]

Let $R=\left(r_{1}, r_{2}, \ldots, r_{m}\right)$ be any partition of length $m$. If $E$ is ample, then $H^{n, q}\left(X, \mathcal{S}_{R} E \otimes(\operatorname{det} E)^{m}\right)=0$ for $q>0$.

Note that this theorem can be derived from that of Griffiths, as follows:

Let $R=\left(r_{1}, \ldots, r_{m}\right)$ be a partition of length $m$, and $r=r_{1}+\ldots+r_{m}$

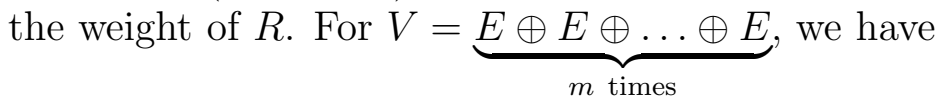

$\mathcal{S}_{R} E \otimes(\operatorname{det} E)^{m} \subset S^{r_{1}} E \otimes S^{r_{2}} E \otimes \ldots \otimes S^{r_{m}} E \otimes(\operatorname{det} E)^{m} \subset S^{r} V \otimes$ $\operatorname{det} V$. Then we use Theorem 2.5.

An extention of the last result to the whole Dolbeault cohomology is due to Manivel [M2].

For $E$ ample and arbitrary partition $\lambda$ the question of finding an exact condition for $H^{p, q}\left(X, \mathcal{S}_{\lambda} E\right)$ to vanish is still open. For a precise conjecture in the case $p=n$ see [L].

In [LN], as a special case of a more general result, this conjecture is proved for any $(p, q)$ and any hook Schur functor $\Gamma_{k}^{\alpha} E$.

The latter are defined for $0 \leq \alpha<k$ and correspond to the partition $(\alpha+1,1, \ldots, 1)$ of weight $k \in \mathbb{N}^{*}$. Inductively, they can be defined as 
follows:

$$
\Gamma_{k}^{0} E=\wedge^{k} E
$$

and

$$
\wedge^{k-\alpha} E \otimes S^{\alpha} E=\Gamma_{k}^{\alpha-1} E \oplus \Gamma_{k}^{\alpha} E
$$

for $0<\alpha<k$. In particular, $\Gamma_{k}^{k-1} E=S^{k} E$. Note that $\Gamma_{k}^{\alpha} E=0$ for $d-k+\alpha<0$.

Define a function $\delta: \mathbb{N} \rightarrow \mathbb{N}^{*}$ by:

$$
\left(\begin{array}{c}
\delta(x) \\
2
\end{array}\right) \leq x<\left(\begin{array}{c}
\delta(x)+1 \\
2
\end{array}\right)
$$

In other words,

$$
\begin{aligned}
& \delta(0)=1 \\
& \delta(1)=\delta(2)=2 \\
& \delta(3)=\delta(4)=\delta(5)=3 \\
& \delta(6)=\delta(7)=\delta(8)=\delta(9)=4
\end{aligned}
$$

etc...

Theorem 2.7. If $E$ is ample, then $H^{p, q}\left(X, \Gamma_{k}^{\alpha} E\right)=0$ for

$$
q+p-n>(\delta(n-p)+\alpha)(d-k+2 \alpha)-\alpha(\alpha+1) \text {. }
$$

For $\alpha=k-d$ one obtains $H^{p, q}\left(X, S^{\alpha} E \otimes \operatorname{det} E\right)=0$, when $p+q-n>$ $(\delta(n-p)-1)(k-d)$. For $p=n$, this specializes to Griffiths' vanishing theorem.

In the present paper we prove an extension to Schur functors of Le Potier's theorem (Theorem 2.3).

\section{BASICS DEFINITIONS AND TOOLS}

\subsection{Some notations and definitions.}

$\mathbb{N}^{*}=\mathbb{N}-\{0\}, \quad I(r)=\{1,2, \ldots, r\} \subset \mathbb{N}^{*}$.

For $(i, j) \in \mathbb{Z} \times \mathbb{Z}$, we call $i$ the height and $j$ the width of $(i, j)$. For $S \subset I(r) \times \mathbb{Z}, \operatorname{card}(S)<\infty$, we define the sequence

$$
[S]: I(r) \longrightarrow \mathbb{N} \text { by }[S]_{i}=\operatorname{card}\{j \in \mathbb{Z} \mid(i, j) \in S\}
$$

For $S \subset I(r) \times \mathbb{N}^{*}, \operatorname{card}(S)<\infty$, we define

$$
\langle S\rangle: \mathbb{N}^{*} \rightarrow \mathbb{N} \text { by }\langle S\rangle_{j}=\operatorname{card}\{i \in I(r) \mid(i, j) \in S\} .
$$

Partitions $u$ of lenght $l(u) \leq r$ are weakly decreasing sequences $u$ : $I(r) \rightarrow \mathbb{N}$. More precisely, we regard partitions $u=\left(u_{1}, u_{2}, \ldots, u_{r}\right)$ and $\left(u_{1}, u_{2}, \ldots, u_{r}, 0, \ldots, 0\right)$ as equivalent. For $u_{r} \neq 0$ the length of $u$ is $r$. 
The weight $u$ is given by

$$
|u|=\operatorname{card}(Y(u))
$$

where

$$
Y(u)=\left\{(i, j) \in I(r) \times \mathbb{N}^{*} \mid 1 \leq j \leq u_{i}\right\}
$$

is the Young diagram of $u$. Equivalently, $|u|=\sum_{i} u_{i}$.

For example the Young diagram of $u=(4,2,1,0)$ is

$$
Y(u)=\{(1,1),(1,2),(1,3),(1,4),(2,1),(2,2),(3,1)\},
$$

the length is 3 and the weight is $|u|=7$.

The transpose $\tilde{u}$ of a partition $u$ is defined by

$$
Y(\tilde{u})=\widetilde{Y(u)}
$$

where $\widetilde{(i, j)}=(j, i)$ for $(i, j) \in \mathbb{N}^{*} \times \mathbb{N}^{*}$. For non positive integers $j$ we put $\tilde{u}_{j}=+\infty$.

We define the squared norm of a partition $u$ by $\|u\|^{2}=\sum_{i \in \mathbb{N}^{*}} \tilde{u}_{i}^{2}$.

When $\psi$ is a map $I(r) \rightarrow \mathbb{Z}$, we denote the corresponding weakly decreasing sequence by $\psi^{\geq}$. More precisely,

$$
\psi^{\geq}=\psi \circ \sigma
$$

where $\sigma$ is any permutation of $I(r)$ such that $\psi \circ \sigma$ is weakly decreasing. When the image of $\psi$ lies in $\mathbb{N}^{*}, \psi^{\geq}$is a partition of length $r$.

When $u$ is a weakly decreasing sequence, let $u^{>}$be the sequence obtained by removing repetitions, in other words the strictly decreasing sequence which has the same set of terms as $u$. For a finite sequence $u$ let $u^{<}$be the strictly increasing sequence which has the same set of terms as $u$.

Every partition $u$ can be reconstructed from $a=u^{>}$and $s=\tilde{u}^{<}$. We write $u=a_{s}$. Explicitly

$$
a_{s}=(\underbrace{a_{1}, \ldots, a_{1}}_{s_{1} \text { times }}, \underbrace{a_{2}, \ldots, a_{2}}_{\left(s_{2}-s_{1}\right) \text { times }}, \ldots, \underbrace{a_{j}, \ldots, a_{j}}_{\left(s_{j}-s_{j-1}\right) \text { times }}, \ldots) .
$$

The latter notation also will be used for general sequences $a$ of finite length.

For each partition $u$ one has a Schur functor $\mathcal{S}_{u}: \mathcal{V} \rightarrow \mathcal{V}$, where $\mathcal{V}$ is the category of complex vector spaces and linear maps. If $u_{r}>0$ for some $r>\operatorname{dim} V$, one has $\mathcal{S}_{u} V=0$.

By functoriality $\mathcal{S}_{u}$ also operates on vector bundles over a given manifold $X$. 
A generalized partition of length $r$ is a weakly decreasing sequence $u: I(r) \rightarrow \mathbb{Z}$.

We define the diagram of a generalized partition $u$ by

$$
\mathcal{D}(u)=\left\{(i, j) \in I(r) \times \mathbb{Z} \mid j \leq u_{i}\right\} .
$$

Note that this diagram is infinite even when $u$ is a usual partition.

\section{Example}

For the generalized partition $u=(2,1,0,-1,-2), \mathcal{D}(u)$ is the set of the following marked points :

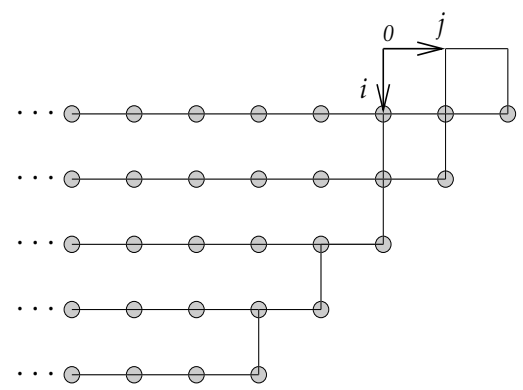

FIGURE 1. diagram of a partition

We define the involution

$$
\chi^{*}: I(r) \times \mathbb{Z} \rightarrow I(r) \times \mathbb{Z} \text { by } \chi^{*}(i, j)=(r+1-i, 1-j),
$$

and the reversed generalized partition $\chi(u)$ by

$$
\mathcal{D}(\chi(u))^{c}=\chi^{*}(\mathcal{D}(u))
$$

where ()$^{c}$ denotes the complement in $I(r) \times \mathbb{Z}$. Explicitly

$$
\chi(u)=\left(-u_{r}, \ldots,-u_{2},-u_{1}\right) \text { for } u=\left(u_{1}, u_{2}, \ldots, u_{r}\right) .
$$

For the category $\mathcal{V}_{r}$ of complex vector spaces of dimension $r$ we extend the Schur functor notation $\mathcal{V}_{r} \rightarrow \mathcal{V}$ to generalized partitions of length $r$ by

$$
\mathcal{S}_{u-\mathbf{k}_{r}} V=\mathcal{S}_{u} V \otimes\left(\operatorname{det} V^{*}\right)^{k}, \quad k \in \mathbb{N}^{*},
$$

where $\mathbf{k}_{r}$ is the partition $(k, k, \ldots, k)$ of length $r$ and $V$ is a complex vector space of dimension $r$.

For $u=\left(u_{1}, u_{2}, \ldots, u_{r}\right)$ we have

$$
\mathcal{S}_{u} V^{*} \simeq \mathcal{S}_{\chi(u)} V
$$

If a sequence $u$ is not weakly decreasing, we put $\mathcal{S}_{u} V=0$. 
If $\mathcal{D}(v) \subset \mathcal{D}(u)$, we say that the pair $u, v$ forms a skew partition $u / v$. We define the diagram of such a skew partition by

$$
\mathcal{D}(u / v)=\mathcal{D}(u) / \mathcal{D}(v)
$$

where $\mathcal{D}(u) / \mathcal{D}(v)$ denotes difference of sets, and the weight by

$$
|u / v|=\operatorname{card}(\mathcal{D}(u / v))
$$

The reversed skew partition is given by

$$
\chi(u / v)=\chi(v) / \chi(u)
$$

\subsection{On the dominance partial order and ampleness.}

Definition 3.3. Let $I=\left(i_{1}, i_{2} \ldots\right), J=\left(j_{1}, j_{2} \ldots\right)$ be partitions of the same weight. We say that

$$
I \succeq J \quad \text { if } \quad \sum_{k=1}^{l} i_{k} \geq \sum_{k=1}^{l} j_{k} \text { for any } l .
$$

This relation is called the dominance partial order.

Definition 3.4. For partitions $I, J$ of arbitrary weight, this definition is generalized in [LN] by $I \preceq J$ for $|J| I \preceq|I| J$. Here the multiplication of a partition $I$ by $n \in \mathbb{N}$ is defined by $n\left(i_{1}, i_{2}, \ldots\right)=\left(n i_{1}, n i_{2}, \ldots\right)$.

\section{Example}

For the partition of weight $5 I=(1,1,1,1,1)$, and $J=(2,1)$ of weight 3 , we have $I \preceq J$ because $1 / 5<2 / 3,2 / 5<1,3 / 5<1$ and $4 / 5<1$.

The following lemma of Macdonald concern partitions of the same weight, but his proof can be adapted easily to partitions of arbitrary weight.

Lemma 3.5. $[M, p .7]$

For any non-trivial partitions of arbitrary weight $I, J$

$$
I \preceq J \Longleftrightarrow \tilde{I} \succeq \tilde{J} .
$$

In $[\mathrm{LN}]$ we also proved

Lemma 3.6. $[L N]$

For any partitions $I$ and $J$ :

$$
\text { if } I \succeq J \text {, then } \mathcal{S}_{I} E \text { ample } \Longrightarrow \mathcal{S}_{J} E \text { ample. }
$$


In particular,

$$
\text { if } I \simeq J \text { then } \mathcal{S}_{I} E \text { ample } \Longleftrightarrow \mathcal{S}_{J} E \text { ample. }
$$

We write $I \simeq J$ if $I \succeq J$ and $I \preceq J$. For example $(k, 0,0, \ldots) \simeq$ $(1,0,0, \ldots)$

\section{ON THE LitTleWOOD-RichaRdson RULES}

Definition 4.1. On any skew partition $w / u$, we define on $\mathcal{D}(w / u)$ the Littlewood-Richardson (LR) order by

$$
\begin{aligned}
& (i, j)<_{L R}\left(i^{\prime}, j^{\prime}\right) \text { for } \quad i<i^{\prime} \\
& (i, j)<_{L R}\left(i, j^{\prime}\right) \text { for } \quad j>j^{\prime} .
\end{aligned}
$$

In this section $w, u$ are fixed and $x<_{L R} y$ implies $x, y \in \mathcal{D}(w / u)$.

Let $w / u$ be a skew partition, and $v=\left(v_{1}, \ldots, v_{r}\right)$ a partition such that $|v|=|w / u|$. We denote $c_{1}$ the numbering

$c_{1}: \mathcal{D}(w / u) \rightarrow \mathbb{N}^{*}$ with $\operatorname{card}\left\{x \in \mathcal{D}(w / u) \mid c_{1}(x)=k\right\}=v_{k}, \forall k \in \mathbb{N}^{*}$

Definition 4.2. The numbering $c_{1}$ is said to satisfy the LR rules, iff

$\left(L_{1}\right): c_{1}$ is strictly increasing on each column of $\mathcal{D}(w / u)$,

$\left(L_{2}\right): c_{1}$ is weakly increasing on each row of $\mathcal{D}(w / u)$,

$\left(L_{3}\right)$ : for all $x \in \mathcal{D}(w / u)$ and all $k \in \mathbb{N}^{*}$, one has $\sigma_{k}(x) \geq \sigma_{k+1}(x)$, where

$$
\sigma_{k}(x)=\operatorname{card}\left\{y \leq_{L R} x \mid c_{1}(y)=k\right\}
$$

Definition 4.3. For a given $c_{1}$, let $c_{2}: \mathcal{D}(w / u) \rightarrow \mathbb{N}^{*}$ with $c_{2}(x)=$ $\operatorname{card}\left\{y \leq_{L R} x \mid c_{1}(y)=c_{1}(x)\right\}$.

Then $c=\left(c_{1}, c_{2}\right), c: \mathcal{D}(w / u) \stackrel{\sim}{\longrightarrow} Y(v)$ is a bijection. Let $b=c^{-1}$, with $b=\left(b_{1}, b_{2}\right)$.

Lemma 4.4. The map $b$ is a strictly increasing function of the width on each row of $Y(v)$ with respect to the order $<_{L R}$.

Proof:

Let $x=(i, j), y=\left(i, j^{\prime}\right), j>j^{\prime}$. Then $\operatorname{card}\left\{z \leq_{L R} x \mid c_{1}(z)=i\right\}=$ $j>j^{\prime}=\operatorname{card}\left\{z \leq_{L R} y \mid c_{1}(z)=i\right\}$, thus $b(i, j)>_{L R} b\left(i, j^{\prime}\right)$.

We put $C(x)=\left\{c(y) \mid y \leq_{L R} x\right\}$.

Remark 4.5. $(i, j) \in C(x) \Longleftrightarrow b(i, j) \leq_{L R} x$. By the previous lemma, $i, j \in \mathbb{N}^{*},(i, j) \in C(x) \Longleftrightarrow j \leq \operatorname{card}\left\{y \leq_{L R} x \mid c_{1}(y)=i\right\}=\sigma_{i}(x)$.

Lemma 4.6. Assume that $c_{1}$ satisfies the $L R$ conditions for each $x \in$ $\mathcal{D}(w / u)$. Then all $C(x)$ are Young diagrams. 


\section{Proof:}

We have to show $(i, j) \in C(x) \Longrightarrow\left(i^{\prime}, j\right) \in C(x)$ for $i^{\prime}=1, \ldots, i-1$, and $(i, j) \in C(x) \Longrightarrow\left(i, j^{\prime}\right) \in C(x)$ for $j^{\prime}=1, \ldots, j-1$. The first implication follows from

$j \leq \sigma_{i}(x) \leq{ }^{\left(L_{3}\right)} \sigma_{i^{\prime}}(x)$. The superscript over the inequality sign indicates that this inequality holds by virtue of the corresponding property.

The second implication follows from remark 4.5.

Definition 4.7. We say that the set $C(x)$ satisfies the condition $(Y)$, If for each $x \in \mathcal{D}(w / u), C(x)$ is a Young diagram.

Lemma 4.8. Let $c=\left(c_{1}, c_{2}^{\prime}\right), c: \mathcal{D}(w / u) \longrightarrow Y(v)$ be a bijection, such that $(Y)$ is true and $c_{1}$ satisfies $\left(L_{1}\right),\left(L_{2}\right)$. Then $c_{1}$ satisfies the $L R$ rules and $c_{2}^{\prime}=c_{2}$.

Proof:

The first part of the conclusion is obvious, since $C(x)$ is a Young diagram, and the length of the $k$-th row of $C(x)$ is equal to $\sigma_{k}(x)$.

The restriction of $c_{2}^{\prime}$ to the set

$$
\left\{x \in \mathcal{D}(w / u) \mid c_{1}(x)=i\right\}
$$

is an order-preserving map to the $i$-th row of $Y(v)$, where the latter is ordered by the width, Indeed

For $x, y \in \mathcal{D}(w / u)$ with $c_{1}(x)=c_{1}(y)=i$, we have $x=b(c(x))=$ $b\left(i, c_{2}^{\prime}(x)\right)$, and $y=b\left(i, c_{2}^{\prime}(y)\right)$, then $c_{2}^{\prime}(x)<c_{2}^{\prime}(y) \Longleftrightarrow x<_{L R} y$ by the Lemma 4.7.

Since both sets have $v_{i}$ elements, this map is the unique orderpreserving bijection.

From now on we will use the

Definition 4.9. We say that a bijection $c: \mathcal{D}(w / u) \stackrel{\sim}{\longrightarrow} Y(v)$ satisfies the LR rules, iff $\left(L_{1}\right),\left(L_{2}\right)$ of the definition 4.2 and $(Y)$ are satisfied.

Recall that $b=c^{-1}$.

The importance of the LR rules is due to the following well-known proposition.

Proposition 4.10. Let $\operatorname{dim} V=r, \quad u$ a generalized partition of length $r$ and $u^{\prime}$ a partition. One has

$$
\mathcal{S}_{u} V \otimes \mathcal{S}_{u^{\prime}} V \simeq \bigoplus_{\substack{(w, b) \in L R\left(u, u^{\prime}\right) \\ 10}} \mathcal{S}_{w} V
$$


where $L R\left(u, u^{\prime}\right)$ consists of pairs $(w, b)$ such that $w / u$ is a skew partition and

$$
b: Y\left(u^{\prime}\right) \stackrel{\sim}{\longrightarrow} \mathcal{D}(w / u)
$$

a bijection satisfying the Littlewood-Richardson rules.

The LR rules have a useful symmetry which is hidden in their original definition, but will be made explicit in the following proposition.

Proposition 4.11. c satisfies the LR rules, iff

(h): On each column of $\mathcal{D}(w / u), c_{1}$ preserves the order of the heights

(th): On each column of $\mathcal{D}(w / u), c_{2}$ weakly inverts this order (w): On each row of $\mathcal{D}(w / u), c_{2}$ inverts the order of the widths

(tw): On each row of $\mathcal{D}(w / u), c_{1}$ weakly preserves this order

(h' ): On each column of $Y(v), b_{1}$ preserves the order of the heights

(th'): On each column of $Y(v), b_{2}$ weakly inverts this order

$\left(\mathbf{w}^{\prime}\right)$ : On each row of $Y(v), b_{2}$ inverts the order of the widths

(tw'): On each row of $Y(v), b_{1}$ weakly preserves this order.

\section{Proof:}

Conditions (h), (tw) are restatements of $\left(L_{1}\right),\left(L_{2}\right)$, and $\left(\mathrm{tw}^{\prime}\right)$ follows from lemma 4.4 .

To show $\left(L_{1}, L_{2}, Y\right) \Longrightarrow($ th $)$ we show $\left(L_{1}, L_{2}, L_{3}\right) \Longrightarrow($ th $)$, which is equivalent by the lemmas 4.6 and 4.8 .

Let $x, y, x^{\prime}, y^{\prime} \in \mathcal{D}(w / u)$ with $y=(i-1, j), x=(i, j)$

and $y^{\prime}=(i-1, j-1), x^{\prime}=(i, j-1)$, with $c_{1}(y)=k, c_{1}(x)=k^{\prime}$, by $\left(L_{1}\right), k<k^{\prime}$. We have $\sigma_{k}(x) \leq \sigma_{k}(y)$.

Consider the case

a) $\sigma_{k}(x)=\sigma_{k}(y)$

This case corresponds to $y^{\prime} \notin \mathcal{D}(w / u)$ or $c_{1}\left(y^{\prime}\right)<c_{1}(x)-1$. We have by $\left(L_{3}\right) \sigma_{k^{\prime}}(x) \leq \sigma_{k}(x)$ hence $\sigma_{k^{\prime}}(x) \leq \sigma_{k}(y)$, which is by definition $c_{2}(x) \leq c_{2}(y)$.

b) $\sigma_{k}(x)>\sigma_{k}(y)$

This case corresponds to $y^{\prime} \in \mathcal{D}(w / u)$ and $c_{1}\left(y^{\prime}\right) \geq c_{1}(x)-1$.

Moreover

$c_{1}\left(y^{\prime}\right)<^{(h)} c_{1}\left(x^{\prime}\right) \leq^{(t w)} c_{1}(x)$ and $c_{1}\left(y^{\prime}\right) \leq^{(t w)} c_{1}(y)<^{(h)} c_{1}(x)$.

This implies

$c_{1}(x)=c_{1}\left(x^{\prime}\right)$ and $c_{1}\left(y^{\prime}\right)=c_{1}(y)=c_{1}(x)-1$.

This gives $\sigma_{k^{\prime}}(x)=\sigma_{k^{\prime}}\left(x^{\prime}\right)-1$ and $\sigma_{k}(y)=\sigma_{k}\left(y^{\prime}\right)-1$.

Now we use the induction on $j$, so that we can assume $c_{2}\left(x^{\prime}\right) \leq c_{2}\left(y^{\prime}\right)$. Thus

$$
c_{2}(x)=\sigma_{k^{\prime}}(x)=c_{2}\left(x^{\prime}\right)-1 \text { and } c_{2}(y)=\sigma_{k}(y)=c_{2}\left(y^{\prime}\right)-1 .
$$


The starting step of the induction is when $x$ and $y$ are such that $y^{\prime} \notin \mathcal{D}(w / u)$, which is the case of a).

To prove $(\mathrm{tw}),(\mathrm{Y}) \Longrightarrow(\mathrm{w})$, assume $(i, j),(i, j+1) \in \mathcal{D}(w / u)$. We want to show that the assumption $c_{2}(i, j+1) \geq c_{2}(i, j)$ leads to a contradiction.

Now $c_{1}(i, j+1) \geq^{(t w)} c_{1}(i, j)$, and the two inequalities together imply that $c(i, j)$ belongs to the Young diagram $C((i, j+1))$, which is wrong, since $(i, j)>_{L R}(i, j+1)$.

To prove $\left(\mathrm{h}^{\prime}\right)$, assume that $i^{\prime}<i, b_{1}\left(i^{\prime}, j\right) \geq b_{1}(i, j)$.

The case $b_{1}\left(i^{\prime}, j\right)=b_{1}(i, j)$ is excluded by $(\mathrm{w})$, which just has been proved. For $b_{1}\left(i^{\prime}, j\right)>b_{1}(i, j)$, we have $\left(i^{\prime}, j\right) \notin C(b(i, j)),(i, j) \in$ $C(b(i, j))$, which contradicts $(\mathrm{Y})$.

To prove $\left(\mathrm{h}^{\prime}\right),(\mathrm{w}),(\mathrm{th}) \Longrightarrow\left(\mathrm{th}^{\prime}\right)$ assume for $x=(i, j), x^{\prime}=\left(i^{\prime}, j\right)$, $x, x^{\prime} \in Y(v)$ that $i^{\prime}<i, b_{2}(x)>b_{2}\left(x^{\prime}\right)$. Let $z=\left(b_{1}(x), b_{2}\left(x^{\prime}\right)\right)$. Since $b_{1}(x)>b_{1}\left(x^{\prime}\right)$ by $\left(\mathrm{h}^{\prime}\right)$ we have $z \in \mathcal{D}(w / u)$. This yields

$$
j=c_{2}(b(x)) \stackrel{(w)}{<} c_{2}(z) \stackrel{(t h)}{\leq} c_{2}\left(b\left(x^{\prime}\right)\right)=j \text {, which is absurd. }
$$

To prove $(\mathrm{h}),(\mathrm{tw}),(\mathrm{Y}) \Longrightarrow\left(\mathrm{w}^{\prime}\right)$ let $\left(i, j^{\prime}\right),(i, j) \in Y(v), j^{\prime}<j$. By lemma 4.4 we have $b_{1}\left(i, j^{\prime}\right) \leq b_{1}(i, j)$.

In the case $b_{1}\left(i, j^{\prime}\right)=b_{1}(i, j)$, we have $b_{2}(i, j)<b_{2}\left(i, j^{\prime}\right)$, by lemma 4.4 , thus $\left(\mathrm{w}^{\prime}\right)$. To prove $\left(\mathrm{w}^{\prime}\right)$ in the case $b_{1}\left(i, j^{\prime}\right)<b_{1}(i, j)$, assume $b_{2}\left(i, j^{\prime}\right) \leq b_{2}(i, j)$. Then $z \in \mathcal{D}(w / u)$, where $z=\left(b_{1}(i, j), b_{2}\left(i, j^{\prime}\right)\right)$.

Thus $i=c_{1}\left(b\left(i, j^{\prime}\right)\right) \stackrel{(h)}{<} c_{1}(z) \stackrel{(t w)}{\leq} c_{1}(b(i, j))=i$, which is absurd.

Finally let us show $\left(\mathrm{h}^{\prime}\right),\left(\mathrm{w}^{\prime}\right),\left(\mathrm{tw}^{\prime}\right) \Longrightarrow(\mathrm{Y})$. The implication

$(i, j) \in C(x), j^{\prime}<j \Longrightarrow\left(i, j^{\prime}\right) \in C(x)$ is equivalent to

$\left(j^{\prime}<j \Longrightarrow b\left(i, j^{\prime}\right)<_{L R} b(i, j)\right)$.

For $b_{1}\left(i, j^{\prime}\right)=b_{1}(i, j)$ this follows from $\left(\mathrm{w}^{\prime}\right)$ (or from (w).) The case $b_{1}(i, j)<_{L R} b_{1}\left(i, j^{\prime}\right)$ is excluded by $\left(\mathrm{tw}^{\prime}\right)$.

The implication

$(i, j) \in C(x), i^{\prime}<i \Longrightarrow\left(i^{\prime}, j\right) \in C(x)$ is equivalent to

$\left(i^{\prime}<i \Longrightarrow b\left(i^{\prime}, j\right)<_{L R} b(i, j)\right)$.

By $\left(\mathrm{h}^{\prime}\right)$ one even has $b_{1}\left(i^{\prime}, j\right)<b_{1}(i, j)$.

Remark 4.12. Clearly the conditions (h, $\left.\mathrm{w}, \mathrm{th}, \mathrm{tw}, \mathrm{h}^{\prime}, \mathrm{w}^{\prime}, \mathrm{th}^{\prime}, \mathrm{tw}^{\prime}\right)$ are not independent. For example, we have proved $\left(\mathrm{h}, \mathrm{tw}, \mathrm{w}^{\prime}, \mathrm{h}^{\prime}, \mathrm{tw}^{\prime}\right) \Rightarrow$ $(\mathrm{h}, \mathrm{tw}, \mathrm{Y}) \Rightarrow\left(\mathrm{w}, \mathrm{th}, \mathrm{th}^{\prime}\right)$.

Remark 4.13. The set of conditions remains invariant under the replacement 


$$
\left.Y(v) \rightarrow Y(\tilde{v}), \quad \text { and } \quad \mathcal{D}(w / u) \rightarrow \chi^{*}(\widetilde{\mathcal{D}(w / u})\right)
$$

which exchanges columns and rows.

Definition 4.14. For $U \subseteq \mathbb{Z} \times \mathbb{Z}$, a map

$b: U \longrightarrow \mathbb{Z} \times \mathbb{Z}$ with $b(i, j)=\left(b_{1}(i, j), b_{2}(i, j)\right)$ is called height increasing if $b_{1}(i, j) \geq i, \forall(i, j) \in U$

Remark 4.15. For partitions $u, v$ of the same weight, the dominance partial order can be characterized by the property that $u \preceq v$ if there is a height increasing bijection $b: Y(v) \longrightarrow Y(u)$.

Lemma 4.16. If $A \subset \mathbb{N}^{*} \times \mathbb{N}^{*}$ and $b: A \longrightarrow \mathbb{N}^{*} \times \mathbb{N}^{*}$ preserves the order of the height on each column of $A$, then $b$ is height increasing.

Proof:

By induction, since $1 \leq b_{1}(1, j)<b_{1}(2, j)<\ldots<b_{1}(i, j)$ for all $(i, j) \in A$. This implies $b_{1}(2, j) \geq 2$ etc ...

\section{Cohomology groups on Flag manifolds}

For $V$ a vector space of dimension $d$ and a sequence $s=\left(s_{0}, s_{1}, \ldots, s_{l}\right)$ such that $0=s_{0}<s_{1}<s_{2}<\ldots<s_{l}<d$, the flag manifold $\mathcal{F} l_{s}(V)=\mathcal{F} l_{s_{1}, s_{2}, \ldots, s_{l}}(V)$ given by subspaces $V_{s_{i}} \subset V$ of codimension $s_{i}$ has natural vector bundles $Q_{i}$ with fibers $V_{s_{i-1}} / V_{s_{i}}$.

For a partition $a=\left(a_{1}, a_{2}, \ldots, a_{l}\right)$ such that $a_{1}>a_{2}>\ldots>a_{l}$, we consider the Schur type line bundle

$$
Q^{a}=\bigotimes_{k=1}^{l} \operatorname{det}\left(Q_{k}\right)^{a_{k}}
$$

Our aim is to prove

\section{Theorem 5.1.}

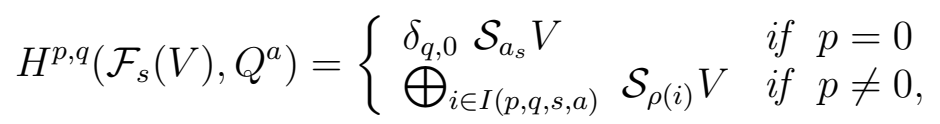

where $|\rho(i)|=\left|a_{s}\right|$ and for all $i$ in the index set $I(p, q, s, a)$,

(i):

$$
\rho(i) \prec a_{s}
$$

(ii):

$$
p+q+1+\left\|a_{s}\right\|^{2} \leq\|\rho(i)\|^{2} .
$$


The main tool for deriving such results is

Bott's Theorem : [ B ] or [D2]

Let $V$ be a complex vector space of dimension $d$ and $\mathcal{F}(V)$ the complete flag manifold of $V$. Let $a \in \mathbb{Z}^{d}$ and $I(d)=(1,2, \ldots, d)$.

Define $\psi(a)=(a-I(d))^{\geq}+I(d)$, where $(a-I(d))^{\geq}$is the sequence obtained by rearranging the terms of $(a-I(d))$ in weakly decreasing order.

We call $i(a)$ the number of strict inversions of $(a-I(d))$ :

$i(a)=\operatorname{card}\left\{(i, j) \mid i<j,(a-I(d))_{i}<(a-I(d))_{j}\right\}$.

Then

$$
H^{q}\left(\mathcal{F}(V), Q^{a}\right)=\delta_{q, i(a)} \mathcal{S}_{\psi(a)} V
$$

Recall that one puts $\mathcal{S}_{\psi(a)} V=0$ if $\psi(a)$ is not a partition. In particular the cohomology of $Q^{a}$ is non vanishing iff all components of $a-I(d)$ are pairwise distinct.

Definition 5.2. For $a \in \mathbb{Z}^{d}$, we say that $a$ is admissible iff all components of $a-I(d)$ are pairwise distinct.

Corollary 5.3. For the incomplete flag $\mathcal{F} l_{s}(V)$, we have [M1]

$$
H^{q}\left(\mathcal{F} l_{s}(V), Q^{a}\right)=\delta_{q, i(a)} \mathcal{S}_{\psi\left(a_{s}\right)} V .
$$

Let $G_{r}(V)$ be the Grasmannian manifold of codimension $r$ subspaces of $V, Q$ and $S$ the universal quotient bundle and the universal subbundle on this manifold. Then the following statement holds:

Corollary 5.4. For $u, v$ generalized partitions of lengths $r$ and $d-r$ respectively, we have [M1]

$$
H^{q}\left(G_{r}(V), \mathcal{S}_{u} Q \otimes \mathcal{S}_{v} S\right)=\left\{\begin{array}{cr}
\mathcal{S}_{\psi(u, v)} V & \text { if } q=i(u, v), \\
0 & \text { otherwise. }
\end{array}\right.
$$

Note that $|\psi(u, v)|=|u|+|v|$.

Let $w$ be a generalized partition of length $r$ and $u$ a partition, let $a=$ $(w, \tilde{u})$, then the set of elements in $(a-I(d))$ is $\left\{\left\{\alpha_{i}\right\}_{i=1, \ldots, r},\left\{\beta_{j}\right\}_{j=1, \ldots, d-r}\right\}$, where $\alpha_{i}=w_{i}-i, \beta_{j}=\tilde{u}_{j}-(r+j)$.

$(w, \tilde{u})$ is admissible iff $\forall(i, j) \in I(r) \times I(d-r), \alpha_{i} \neq \beta_{j}$. We have $i(a)=\operatorname{card}\left\{(i, j) \mid \alpha_{i}<\beta_{j}\right\}$.

Let $[\gamma]$ be the sequence such that, $[\gamma]_{i}=\operatorname{card}\left\{j \mid \alpha_{i}-\beta_{j}<0\right\}$, and $\langle\gamma\rangle$ the sequence such that, $\langle\gamma\rangle_{j}=\operatorname{card}\left\{i \mid \alpha_{i}-\beta_{j}<0\right\}$. 
Lemma 5.5. Let $w$ be a generalized partition of length $r$ and $u a$ partition, such that $(w, \tilde{u})$ is admissible. Define $s_{+}: I(r) \rightarrow \mathbb{Z}$ by $\left(s_{+}\right)_{i}=w_{i}+[\gamma]_{i} \quad$ and $\quad s_{-}: \mathbb{N}^{*} \rightarrow \mathbb{Z} \quad$ by $\quad\left(s_{-}\right)_{j}=\tilde{u}_{j}-\langle\gamma\rangle_{j}$.

Then $s_{+}, s_{-}$are partitions and

with

$$
H^{q}\left(G_{r}(V), S_{w} Q \otimes \wedge_{u} S\right)=\delta_{q, i(w, \tilde{u})} \mathcal{S}_{\psi(w, \tilde{u})} V
$$

(i): $\psi(w, \tilde{u})=\left(s_{+}, s_{-}\right)^{\geq}$,

(ii): $i(w, \tilde{u})=\left|s_{+}\right|-\sum_{i \in I(r)} w_{i}$.

\section{Proof:}

Since the cohomology group is given by Corollary 5.4, we only need to investigate the combinatorics.

Let $a=(w, \tilde{u})$ be admissible. With the above notations the set of elements in $(a-I(d))$ is $\left\{\left\{\alpha_{i}\right\}_{i=1, \ldots, r},\left\{\beta_{j}\right\}_{j=1, \ldots, d-r}\right\}$, where $\alpha_{i}$ is in position $i$ in $(a-I(d))$ and in position $i+[\gamma]_{i}$ in $(a-I(d))^{\geq}$. Thus the term in this position in $(a-I(d))^{\geq}+I(d)$ is $\alpha_{i}+i+[\gamma]_{i}=\left(s_{+}\right)_{i}$.

Similarly, $\quad \beta_{j}$ is in the position $r+j$ in $a-I(d)$, and in the position $r+j-\langle\gamma\rangle_{j}$ in $(a-I(d))^{\geq}$, such that the term in this position in $(a-I(d))^{\geq}+I(d)$ is $\beta_{j}+r+j-\langle\gamma\rangle_{j}=\left(s_{-}\right)_{j}$.

For admissible $a$ the sequences $s_{+}$and $s_{-}$are weakly decreasing. Indeed,

$$
\begin{aligned}
& \left(s_{+}\right)_{i}-\left(s_{+}\right)_{i+1}=w_{i}-w_{i+1}+\operatorname{card}\left\{j \mid \alpha_{i}>\beta_{j}>\alpha_{i+1}\right\} \text { and } \\
& \quad \operatorname{card}\left\{j \in \mathbb{N}^{*} \mid w_{i}-i>\beta_{j}>w_{i+1}-(i+1)\right\} \leq w_{i}-w_{i+1},
\end{aligned}
$$

since $\beta_{j}$ is a strictly decreasing integral sequence, and similarly for $s_{-}$.

Since $s_{-}$converges to 0 , it is a partition. The sequence $s_{+}$has a finite number of terms, which are greater than the limit of $s_{-}$. Thus $s_{+}$is a partition, too.

Finally from $\left(s_{+}\right)_{i}=w_{i}+[\gamma]_{i}$ we get (ii).

For $(w, \tilde{u})$ admissible, we will apply this lemma in the case where $w / \chi(u)$ is a skew partition of length $r$.

In this case we denote by

$$
\begin{aligned}
\Sigma_{ \pm} & =\left\{(i, j) \in \mathcal{D}(w / \chi(u)) \mid \beta_{1-j} \gtrless \alpha_{i}\right\} . \\
& =\left\{(i, j) \in \mathcal{D}(w / \chi(u)) \mid \tilde{u}_{1-j}-(r+1-j) \gtrless w_{i}-i\right\}
\end{aligned}
$$

Note that $(w, \tilde{u})$ is admissible, iff $\mathcal{D}(w / \chi(u))=\Sigma_{+} \cup \Sigma_{-}$. We denote $\mathcal{D}(w / \chi(u))$ by $\Sigma$.

Definition 5.6. We call $\left(w / \chi(u)\right.$ admissible iff $\Sigma=\Sigma_{+} \cup \Sigma_{-}$, and we call $\Sigma_{+}, \Sigma_{-}$the splitting of $\mathcal{D}(w / \chi(u))$ 
Recall that we put $\tilde{u}_{j}=+\infty$ for $j \leq 0$, such that all $(i, j) \in \Sigma$ with $j>0$ belong to $\Sigma_{+}$.

Lemma 5.7. For $(w, \tilde{u})$ admissible and $w / \chi(u)$ a skew partition of length $r$, we have

(i): $s_{+}=\left[\Sigma_{+}\right]$

(ii): $s_{-}=\left\langle\chi^{*}\left(\Sigma_{-}\right)\right\rangle$

(iii): $i(w, \tilde{u})=|u|-\operatorname{card}\left(\Sigma_{-}\right)$.

Proof :

$$
\begin{aligned}
\left(s_{+}\right)_{i} & =w_{i}+\operatorname{card}\left\{j \leq 0 \mid \tilde{u}_{1-j}-1+j-r>w_{i}-i\right\} \\
& =w_{i}+\operatorname{card}\left\{j \leq 0 \mid \beta_{1-j}>\alpha_{i}\right\} .
\end{aligned}
$$

If $j \leq-u_{r+1-i}$ one has $1-j>u_{r+1-i}$, thus $\tilde{u}_{1-j}<r+1-i$. Moreover since $\mathcal{D}(\chi(u)) \subseteq \mathcal{D}(w)$, we have $-u_{r+1-i} \leq w_{i}$. This implies $j \leq w_{i}$, thus $\beta_{1-j}<\alpha_{i}$, and we can write

$$
\left(s_{+}\right)_{i}=w_{i}+\operatorname{card}\left\{j \leq 0 \mid-u_{r+1-i}<j, \beta_{1-j}>\alpha_{i}\right\} .
$$

If $0 \geq j>w_{i}$, we have $1-j \leq u_{r+1-i}$. Thus $\tilde{u}_{1-j} \geq r+1-i$ and $\beta_{1-j}>\alpha_{i}$.

This yields for any $w_{i} \in \mathbb{Z}$

$$
\left(s_{+}\right)_{i}=\operatorname{card}\left\{j \in \mathbb{Z} \mid-u_{r+1-i}<j \leq w_{i}, \beta_{1-j}>\alpha_{i}\right\}=\left[\Sigma_{+}\right]_{i} .
$$

as required.

Similarly we have

$$
\left(s_{-}\right)_{j}=\tilde{u}_{j}-\operatorname{card}\left\{i \in I(r) \mid \beta_{j}>\alpha_{r+1-i}\right\}=\tilde{u}_{j}-\langle\gamma\rangle_{j} .
$$

a) If $u_{i}<j$ we have $\tilde{u}_{j}<i$, and since $\mathcal{D}(\chi(u)) \subseteq \mathcal{D}(w)$, we have $-u_{i} \leq w_{r+1-i}$. this implies $\beta_{j}<\alpha_{r+1-i}$, thus

$\langle\gamma\rangle_{j}=\operatorname{card}\left\{i \in I(r) \mid-u_{i}<1-j, \beta_{j}>\alpha_{r+1-i}\right\}$.

b) If $j \leq-u_{r+1-i}$, we have $j<u_{i}$, thus $\tilde{u}_{j} \geq i$ and $\alpha_{r+1-i}<\beta_{j}$.

Now

$$
\begin{aligned}
\Sigma_{-} & =\left\{i \in I(r) \mid-u_{r+1-i}<j \leq w_{i}, \alpha_{i}>\beta_{1-j}\right\} \\
\chi^{*}\left(\Sigma_{-}\right) & =\left\{i \in I(r) \mid-u_{i}<1-j \leq w_{r+1-i}, \alpha_{r+1-i}>\beta_{j}\right\} \\
\left\langle\chi^{*} \Sigma_{-}\right\rangle_{j} & =\text { card }\left\{i \in I(r) \mid-u_{i}<1-j \leq w_{r+1-i}, \alpha_{r+1-i}>\beta_{j}\right\} \\
& =\text { card }\left\{i \in I(r) \mid-u_{i}<1-j, \alpha_{r+1-i}>\beta_{j}\right\} .
\end{aligned}
$$

The last equality is due to b). Now 


$$
\begin{aligned}
\left\langle\chi^{*} \Sigma_{-}\right\rangle_{j}+\langle\gamma\rangle_{j} & =\text { card }\left\{i \in I(r) \mid-u_{i}<1-j, \alpha_{r+1-i}>\beta_{j}\right\} \\
& +\operatorname{card}\left\{i \in I(r) \mid-u_{i}<1-j \alpha_{r+1-i}<\beta_{j}\right\} \\
& =\text { card }\left\{i \in I(r) \mid-u_{i}<1-j\right\} \\
& =\operatorname{card}\left\{i \in I(r) \mid i \leq \tilde{u}_{j}\right\}=\tilde{u}_{j}
\end{aligned}
$$

as required

Finally $\operatorname{card}\left(\Sigma_{+}\right)+\operatorname{card}\left(\Sigma_{-}\right)=\sum_{i \in I(r)}\left(w_{i}-\chi(u)_{i}\right)$. Together with $\operatorname{card}\left(\Sigma_{+}\right)=\left|s_{+}\right|$and the previous lemma, this yields the desired formula for $i(w, \tilde{u})$.

Lemma 5.8. We have

(i): $\psi(w, \tilde{u})=\left(\left[\Sigma_{+}\right],\left\langle\chi^{*}\left(\Sigma_{-}\right)\right\rangle\right)^{\geq}$

(ii): $\left\langle\Sigma_{-}\right\rangle_{j} \leq\left[\Sigma_{+}\right]_{i} \quad$ for $\quad(i, j) \in \Sigma_{-}$

(iii): $\left[\Sigma_{+}\right]_{i} \leq\left\langle\Sigma_{-}\right\rangle_{j}$ for $\quad(i, j) \in \Sigma_{+}$

Proof:

The preceding lemmas gives (i). We prove ii), the proof of iii) is similar.

Since $\left[\Sigma_{+}\right]_{i}$ is decreasing, it suffices to consider for given $j$

$$
(i, j) \in \Sigma_{-},(i+1, j) \notin \Sigma_{-} .
$$

Determine $j^{\prime}$ such that $\left(i, j^{\prime}\right) \in \Sigma_{-},\left(i, j^{\prime}+1\right) \notin \Sigma_{-}$.

Since $\left(r+1-\tilde{u}_{1-j}, j\right)$ is the element of smallest height in column $j$ of $\Sigma_{-}$and $(i, j)$ the element of greatest height, we have

$\left\langle\Sigma_{-}\right\rangle_{j}=\tilde{u}_{1-j}-r+i \leq \tilde{u}_{1-j^{\prime}}-r+i \leq w_{i}-j^{\prime}=\left[\Sigma_{+}\right]_{i}$.

Since every row of $\mathcal{D}(w, \chi(u))$ contains exactly one row of $\Sigma_{+}$and every column of $u$ yields exactly one column of $\Sigma_{-}$, the length of $\psi(w, \tilde{u})$ is the number of non-vanishing terms of $[\mathcal{D}(w / \chi(u))]$ plus $u_{1}$.

\section{Example}

$$
\begin{aligned}
& w=(5,4,3,2,-1,-2) \\
& u=(7,7,4,3,3,1), \chi(u)=(-1,-3,-3,-4,-7,-7), \\
& \tilde{u}=(6,5,5,3,2,2,2) \\
& \alpha=(4,2,0,-2,-6,-8), \beta=(-1,-3,-4,-7,-9,-10,-11) \\
& {[\gamma]=(0,0,0,0,3,4),\langle\gamma\rangle=(3,2,2,1,0,0,0)} \\
& s_{+}=\left[\Sigma_{+}\right]=(5,4,3,3,2,2), s_{-}=\left\langle\chi^{*} \Sigma_{-}\right\rangle=(3,3,2,2,2,2) .
\end{aligned}
$$

The whole shaded area is $w / \chi(u)$, the set of vertical strips is $\left\langle\chi^{*} \Sigma_{-}\right\rangle$, the set of horizontal strips is $\left[\Sigma_{+}\right]$

Lemma 5.9. Choosing the permutation of shortest length for the reordering of $\left(\left[\Sigma_{+}\right],\left\langle\chi^{*}\left(\Sigma_{-}\right)\right\rangle\right)$yields a natural bijection 


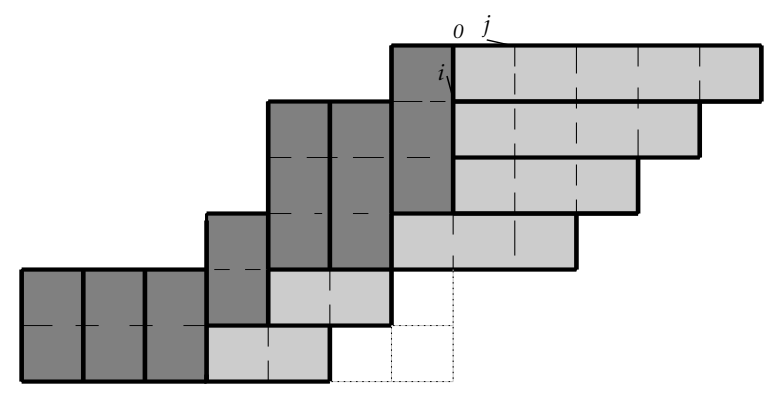

FiguRE 2.

$$
\beta: \Sigma_{+} \cup \Sigma_{-} \stackrel{\sim}{\longrightarrow} Y(\psi(w, \tilde{u})) .
$$

Then $\beta$ is height increasing.

\section{Proof:}

On $\Sigma_{+}$, the bijection $\beta$ is height increasing, since $\left[\Sigma_{+}\right]$is weakly decreasing. For $(i, j) \in \Sigma_{-}$, the inequality derived above implies that the reordering puts $\left\langle\Sigma_{-}\right\rangle_{j}$ after $\left[\Sigma_{+}\right]_{1}, \ldots,\left[\Sigma_{+}\right]_{i}$.

Thus $\beta_{1}(i, j)>i$.

Lemma 5.10. Let $u, v$ be partitions such that $l(u) \leq r$ and $l(v)=r$,

$(w, b) \in L R(\chi(u), v),(w, \tilde{u})$ admissible, and $\rho=\psi(w, \tilde{u})$. Then either

1) $u=0, \rho=v$, or

2) $|u|+i(w, \tilde{u})+1+\|v\|^{2} \leq\|\rho\|^{2}$.

\section{Proof:}

We use induction on $u_{1}+r$, in other words on the length of $\psi(w, \tilde{u})$. For $u_{1}=0$, the statement is obviously true.

Assume $u_{1}>0$. We use the splitting of $\mathcal{D}(w / \chi(u))$ into $\Sigma_{+}, \Sigma_{-}$ introduced above.

Since $v_{r}>0$, we either have $\chi^{*}\left(1, u_{1}\right) \in \Sigma_{-}$or $\chi^{*}\left(1, u_{1}\right) \in \Sigma_{+}$. The two cases will be treated differently.

a) For $\chi^{*}\left(1, u_{1}\right) \in \Sigma_{-}$, consider the partitions $u^{\prime}, v^{\prime}$ given by $\mathcal{D}\left(u^{\prime}\right)=\mathcal{D}(u) \cap\left(I(r) \times u_{1}\right)^{c}$, and $Y\left(v^{\prime}\right)=b^{-1}\left(\mathcal{D}\left(w / \chi\left(u^{\prime}\right)\right)\right)$, in other words if $\tilde{u}=\left(\tilde{u}_{1}, \tilde{u}_{2}, \ldots, \tilde{u}_{u_{1}}\right)$, with $\tilde{u}_{u_{1}}=l$, then $\tilde{u}^{\prime}=\left(\tilde{u}_{1}, \tilde{u}_{2}, \ldots, \tilde{u}_{u_{1}-1}\right)$. We denote by $x_{j}=\left(r+1-j, 1-u_{1}\right), j=1,2, \ldots l$ and $L=\left\{x_{j}, j=\right.$ $1,2, \ldots l\}$

Let $b^{\prime}$ be the restriction of $b$ to $Y\left(v^{\prime}\right)$. By Remark 4.13, $Y\left(v^{\prime}\right)$ is a Young diagram. The Littlewood-Richardson rules yield $\left(w, b^{\prime}\right) \in$ $L R\left(\chi\left(u^{\prime}\right), v^{\prime}\right)$. Note that $u_{1}^{\prime}=u_{1}-1$ and $i(w, \tilde{u})=i\left(w, \tilde{u}^{\prime}\right)$. The skew 
partition $w / \chi\left(u^{\prime}\right)$ is admissible and the splitting of $\mathcal{D}\left(w / \chi\left(u^{\prime}\right)\right)$ is given by $\Sigma_{+}^{\prime}=\Sigma_{+}$, and $\Sigma_{-}=\Sigma_{-}^{\prime} \cup L$

By Lemma 4.5 and Remark 4.13, $Y(v)$ is obtained from $Y\left(v^{\prime}\right)$ by successive unions with the preimages of the set $L$, each union being a Young diagram. Thus we have

$$
\|v\|^{2}-\left\|v^{\prime}\right\|^{2}=\sum_{j=1}^{L}\left(2 c_{1}\left(x_{j}\right)-1\right),
$$

Since $b$ is height increasing ie $c_{1}\left(x_{j}\right) \leq r+1-j$ this yields

$$
\|v\|^{2}-\left\|v^{\prime}\right\|^{2} \leq l(2 r-l) .
$$

The length of $\rho=\psi(w, \tilde{u})$ is $u_{1}+r$. With $\rho^{\prime}=\psi\left(w, \tilde{u}^{\prime}\right)$, lemma 5.9 yields $\rho=\left(\rho^{\prime}, l\right)$ and

$$
\|\rho\|^{2}-\left\|\rho^{\prime}\right\|^{2}=l\left(2\left(u_{1}+r\right)-1\right) .
$$

By Lemma 5.8, we have $l=\left\langle\Sigma_{-}\right\rangle_{-u_{1}} \leq\left[\Sigma_{+}\right]_{r}$.

Since $\left[\Sigma_{+}\right]_{r}>0$ we have $v_{r}^{\prime}>0$ and can use the induction assumption. Thus

$$
\begin{aligned}
|u|+i(w, \tilde{u})+1+\|v\|^{2}-\|\rho\|^{2} & \leq \\
l+l(2 r-l)-l\left(2\left(u_{1}+r\right)-1\right)+1 & =l\left(2-l-2 u_{1}\right)+1 \leq 0,
\end{aligned}
$$

as required.

b) For $\chi^{*}\left(1, u_{1}\right) \in \Sigma_{+}$, the argument is similar. We put

$\mathcal{D}\left(w^{\prime} / \chi\left(u^{\prime}\right)\right)=\mathcal{D}(w / \chi(u)) \cap(\{r\} \times \mathbb{Z})^{c}$, and $Y\left(v^{\prime}\right)=b^{-1} \mathcal{D}\left(w / \chi\left(u^{\prime}\right)\right)$,

in other words if $u=\left(u_{1}, u_{2}, u_{3} \ldots\right)$ then $u^{\prime}=\left(u_{2}, u_{3} \ldots\right)$.

Let $b^{\prime}$ be the restriction of $b$ to $Y\left(v^{\prime}\right)$. The new partitions $v^{\prime}, w^{\prime}$ have length $r^{\prime}=r-1$. The skew partition $w^{\prime} / \chi\left(u^{\prime}\right)$ is admissible and the splitting of $\mathcal{D}\left(w^{\prime} / \chi\left(u^{\prime}\right)\right)$ is given by

$\Sigma_{-}^{\prime}=\Sigma_{-}$. Let $\left[\Sigma_{+}\right]_{r}=l$. We find

$$
\|v\|^{2}-\left\|v^{\prime}\right\|^{2} \leq l(2 r-1),
$$

$\rho=\left(\rho^{\prime}, l\right)$ and

$$
\|\rho\|^{2}-\left\|\rho^{\prime}\right\|^{2}=l\left(2\left(u_{1}+r\right)-1\right) .
$$

Since $\left\langle\Sigma_{-}\right\rangle_{1-u_{1}} \neq 0$, we have $u_{1}^{\prime}=u_{1}$ and $v_{r^{\prime}}^{\prime}>0$, we can use the induction assumption. Altogether 


$$
\begin{aligned}
& |u|+i(w, \tilde{u})+1+\|v\|^{2}-\|\rho\|^{2} \leq \\
& 2 l+l(2 r-1)-l\left(2\left(u_{1}+r\right)-1\right) \\
& =2 l\left(1-u_{1}\right) \leq 0 .
\end{aligned}
$$

\section{Example of the situation of Lemma 5.10 case a)}

For $u=(7,7,4,3,3,1)$ and $v=(9,8,6,5,5,3)$. We have $w / \chi(u)$ on the left hand side the same as the previous example with the same $\left[\Sigma_{+}\right]$, and $\left\langle\chi^{*} \Sigma_{-}\right\rangle$. The shaded area is $\tilde{u}_{u_{1}}$. The partition $v^{\prime}$ is the partition $v$ without the crossing boxes.

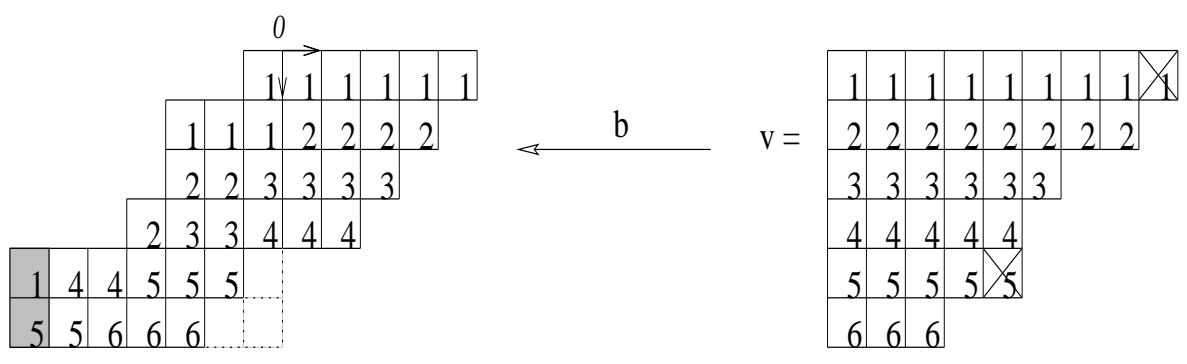

FiguRE 3.

Lemma 5.11. Let $v$ be a partition of length $r$. Then

$$
H^{p, q}\left(G_{r}(V), \mathcal{S}_{v} Q\right)=\bigoplus_{k \in K(p, q, r, v)} \mathcal{S}_{\rho(k)} V,
$$

where $|\rho(k)|=|v|$. We have

(i): $\forall k \in K(p, q, r, v), \rho(k) \preceq v$.

(ii): Moreover, $\rho(k)=v$ only occurs for $p=q=0$, where

$$
\text { for any partition } v \quad H^{0}\left(G_{r}(V), \mathcal{S}_{v} Q\right)=\mathcal{S}_{v} V .
$$

Proof:

It is well known that

$$
\Omega_{G_{r}(V)}^{p}=\wedge^{p}\left(Q^{*} \otimes S\right)=\bigoplus_{u \in \sigma^{p}} \mathcal{S}_{u} Q^{*} \otimes \mathcal{S}_{\tilde{u}} S
$$

where $\sigma^{p}$ is the set of partitions of weight $p$ and length $r$. Then 


$$
\begin{aligned}
H^{p, q}\left(G_{r}(V), \mathcal{S}_{v} Q\right) & =\bigoplus_{u \in \sigma^{p}} H^{q}\left(G_{r}(V), \mathcal{S}_{\chi(u)} Q \otimes \mathcal{S}_{v} Q \otimes \wedge_{u} S\right) \\
& =\bigoplus_{u \in \sigma^{p}} \bigoplus_{(w, b) \in L R(\chi(u), v)} H^{q}\left(G_{r}(V), \mathcal{S}_{w} Q \otimes \wedge_{u} S\right) \\
& =\bigoplus_{u \in \sigma^{p}} \bigoplus_{(w, b) \in L R(\chi(u), v)} \delta_{q, i(w, \tilde{u})} \mathcal{S}_{\psi(w, \tilde{u})} V .
\end{aligned}
$$

For each term on the right-hand side. we have constructed a height increasing bijection

$$
\beta \circ b: Y(v) \stackrel{\sim}{\longrightarrow} Y(\psi(w, \tilde{u})) .
$$

Thus $\psi(w, \tilde{u}) \preceq v$. Since the length of $\psi(w, \tilde{u})$ is at least $u_{1}$ plus the length of $v$, it follows that $\psi(w, \tilde{u})=v$ implies $u=0$, and thus $p=0$.

Corollary 5.12. $H^{(p, q)}\left(G_{r}(V), \operatorname{det} Q\right)=0 \quad$ if $\quad p \neq 0$ or $q \neq 0$,

Proof:

there is no non-trivial partition strictly less than $v=(1,1, \ldots, 1)$ of the same weight as $v$.

This is also a result of Le Potier [LP1,(corol.1)]

In the sequel, we will use the notation $R^{p, q} \mathcal{F}$ for $R^{q} \pi_{*}\left(\Omega^{p} \otimes \mathcal{F}\right)$.

\subsection{Proof of Theorem 5.1.}

For $p=0$, Corollary 1 to Bott's theorem gives $i(a)=0=q$ and $\psi\left(a_{s}\right)=a_{s}$.

For $p \neq 0$ we will use induction on $l$, the length of $s$. Let us consider the Borel-le Potier (B-L) spectral sequence associated to

$$
\pi: Y=\mathcal{F}_{s}(V) \longrightarrow G_{s_{l}}(V)=X .
$$

On $X$ we have the canonical quotient bundle $Q$ with fibres $Q=V / V_{s_{l}}$. The fibres of $Y$ have the form $\mathcal{F} l_{s^{\prime}}\left(Q_{x}\right)$, where $s^{\prime}=\left(s_{1}, \ldots, s_{l-1}\right)$. On $Y$ we have canonical bundles $Q_{i}$ with fibres $V_{s_{i-1}} / V_{s_{i}}$.

As explained in the introduction, the Leray spectral sequence (L) associated to the projection $\pi$, called ${ }^{p}, p \mathcal{E}_{L}$ abuts to the $\mathcal{E}_{1}$ terms of the Borel-Le Potier (BL) spectral sequence:

$$
{ }^{\prime}, p \mathcal{E}_{2, L}^{q-j, j} \stackrel{L}{\Longrightarrow}{ }^{p} \mathcal{E}_{1, B}^{p^{\prime}, q-p^{\prime}} \stackrel{B L}{\Longrightarrow} H^{p, q}\left(\mathcal{F}_{s}(V), Q^{a}\right) .
$$

We have

$$
{ }^{p^{\prime}, p} \mathcal{E}_{2, L}^{q-j, j}=H^{p^{\prime}, q-j}\left(G_{s_{l}}(V), R^{p-p^{\prime}, j} \pi_{*}\left(Q^{a}\right)\right) .
$$


On $Q$ we have flags $\{0\} \subset V_{s_{l-1}} / V_{s_{l}} \subset \ldots V_{s_{1}} / V_{s_{l}} \subset V / V_{s_{l}}$. For $V_{s_{j}} / V_{s_{l}}=V_{s_{j}}^{\prime}$ we have $V_{s_{j-1}}^{\prime} / V_{s_{j}}^{\prime}=Q_{j}$. We rewrite

$$
\begin{aligned}
Q^{a} & =\left(\operatorname{det} Q_{1}\right)^{a_{1}} \otimes \ldots \otimes\left(\operatorname{det} Q_{l}\right)^{a_{l}} \\
& =(\operatorname{det} Q)^{a_{l}} \otimes\left(\operatorname{det} Q_{1}\right)^{a_{1}^{\prime}} \otimes \ldots \otimes\left(\operatorname{det} Q_{l-1}\right)^{a_{l-1}^{\prime}}
\end{aligned}
$$

where $a_{i}^{\prime}=a_{i}-a_{l}$.

Setting $a^{\prime}=\left(a_{1}^{\prime}, \ldots, a_{l-1}^{\prime}\right)$, we have

$$
\begin{aligned}
{ }^{p^{\prime}, p} \mathcal{E}_{2, L}^{q-j, j} & =H^{p^{\prime}, q-j}\left(G_{s_{l}}(V),(\operatorname{det} Q)^{a_{l}} \otimes R^{p-p^{\prime}, j} \pi_{*}\left(Q^{a^{\prime}}\right)\right) \\
& =H^{p^{\prime}, q-j}\left(G_{s_{l}}(V),(\operatorname{det} Q)^{a_{l}} \otimes H^{p-p^{\prime}, j}\left(F_{s_{1}, s_{2} \ldots s_{l-1}}(V), Q^{a^{\prime}}\right)\right) .
\end{aligned}
$$

For $p-p^{\prime}=0$, the desired result follows from Corollary 5.4 with $v=0$ and Lemmas 5.10 and 5.11. For $p^{\prime}<p$ we have by the induction assumption for the graded bundle $(G r)$ associated to the higher direct image,

$$
G r R^{p-p^{\prime}, j} \pi_{*}\left(Q^{a^{\prime}}\right)=\bigoplus_{k \in K} \mathcal{S}_{\rho^{\prime}(k)} Q,
$$

where $\rho^{\prime}(k) \preceq a_{s^{\prime}}^{\prime}$ for all $k \in K$. Consequently,

$$
(\operatorname{det} Q)^{a_{l}} \otimes \mathcal{S}_{\rho^{\prime}(k)} Q=\mathcal{S}_{\rho^{\prime \prime}(k)} Q \quad \text { with } \quad \rho^{\prime \prime}(k) \preceq a_{s} .
$$

Since $\left|\rho^{\prime}(k)\right|=\left|a_{s^{\prime}}^{\prime}\right|$, we have $\left|\rho^{\prime \prime}(k)\right|=\left|a_{s}\right|$.

The bundle $R^{p-p^{\prime}, j} \pi_{*}\left(Q^{a^{\prime}}\right)$ on $X$ is a homogeneous $G l(V)$-bundle, thus specified by a representation of the stabilizer of a point in $X$. Since $G\left(V_{s_{l}}\right)$ acts trivially on the fibres, its representation factorizes through that of $G l(Q)$. By the Schur lemma $[\mathrm{FH}]$, such a representation is reducible and is given by a Schur functor, which implies

$$
R^{p-p^{\prime}, j} \pi_{*}\left(Q^{a^{\prime}}\right) \simeq G r R^{p-p^{\prime}, j} \pi_{*}\left(Q^{a^{\prime}}\right) .
$$

Finally, again by the induction assumption,

$$
p-p^{\prime}+j+1+\left\|a_{s^{\prime}}^{\prime}\right\|^{2} \leq\left\|\rho^{\prime}(k)\right\|^{2}
$$

for all $k$ in the set of subscripts $K$. By Lemma 5.10 we have

$$
p^{\prime}+q-j+\left\|\rho^{\prime}(k)\right\|^{2}+\left|a_{l}\right|^{2} \leq\|\rho(i)\|^{2}
$$

for all $i \in I(p, q, s, a)$. Since $\left\|a_{s}\right\|^{2}=\left\|a_{s^{\prime}}^{\prime}\right\|^{2}+\left|a_{l}\right|^{2}$, the result follows from Lemmas 5.10 and 5.11. 


\section{Proof of Theorems 1.1 And Corollary 1.2}

\subsection{Proof of Theorem 1.1.}

For a partition $R=\left(r_{1}, \ldots, r_{m}\right)$, we take $a=\tilde{R}^{>}, s=\left(s_{1}, \ldots, s_{l}\right)=$ $R^{<}$, such that $a_{s}=\tilde{R}$.

Let $Y=\mathcal{F}_{s_{1}, \ldots, s_{l}}(E)$ and $\mathcal{L}=Q^{a}$ We use induction on $R$ with respect to the dominance partial order.

In the setup discussed in the introduction, we consider the BorelLe Potier spectral sequence given by $X, Y, \mathcal{L}, P$, where $\mathcal{L}=Q^{a}$. Its $\mathcal{E}_{1}$ terms can be evaluated by a Leray spectral sequence, for which

$$
{ }^{p, P} \mathcal{E}_{2, L}^{q-j, j}=H^{p, q-j}\left(X, R^{P-p, j} \pi_{*}\left(Q^{a}\right)\right),
$$

For $P-p=0$, we have $R^{0, j} \pi_{*}\left(Q^{a}\right)=\delta_{j, 0} \wedge_{r} E \quad$ by Theorem 5.1. This implies that for $P-p=0$ the Leray spectral sequence degenerates at $\mathcal{E}_{2}$, and ${ }^{P} \mathcal{E}_{1, B}^{P, q-P}=H^{P, q}\left(X, \wedge_{r} E\right)$.

We have

$$
\begin{aligned}
& P \mathcal{E}_{1, B}^{p-p_{1},(q-1)-\left(p-p_{1}\right)} \stackrel{d_{1, B}}{\longrightarrow} \cdots \stackrel{d_{1, B}}{\longrightarrow} P \mathcal{E}_{1, B}^{p-1,(q-1)-(p-1)} \stackrel{d_{1, B}}{\longrightarrow} P \mathcal{E}_{1, B}^{p, q-p} \stackrel{d_{1, B}}{\longrightarrow} 0 \\
& \ldots
\end{aligned}
$$

In order to show that ${ }^{P} \mathcal{E}_{1, B}^{P, q-P}$ is a subfactor of the limit group $H^{P, q}\left(Y, Q^{a}\right)$, we must prove that the sources of $\stackrel{d_{p_{1}, B}}{\longrightarrow} P \mathcal{E}_{1, B L}^{p, q-p}$ vanish for each $p_{1} \neq 0$.

Now each group $P \mathcal{E}_{1, B}^{p-p_{1},(q-1)-\left(p-p_{1}\right)}$ is a subquotient of

$$
H^{P-p_{1}, q-q_{1}-1}\left(X, R^{p_{1}, q_{1}}\left(Q^{a}\right)\right)
$$

where by Theorem 5.1,

$$
\operatorname{Gr} \mathrm{R}^{\mathrm{p}_{1}, \mathrm{q}_{1}} \pi_{*}\left(\mathrm{Q}^{\mathrm{a}}\right)=\bigoplus \mathcal{S}_{\rho(\mathrm{i})}(\mathrm{E})
$$

with $\rho(i) \prec \tilde{R}$, since $p_{1} \neq 0$. By Lemmas 3.5 and 3.6 the vector bundles $\mathcal{S}_{\rho(i)}(E)$ are ample. Moreover, $p_{1}+q_{1}+1 \leq|\rho|^{2}-|R|^{2}$ by Theorem 5.1. Together with the assumption

$$
P+q-n>\sum_{i=1}^{m} r_{i}\left(d-r_{i}\right)
$$

and $|\rho|=|R|$ this yields $P-p_{1}+q-q_{1}-1-n>\sum_{i=1}^{m} \rho_{i}\left(d-\rho_{i}\right)$. Since $\rho(i) \prec \tilde{R}$ we can use the induction assumption, such that indeed $H^{P-p_{1}, q-q_{1}-1}\left(X, G r R^{p_{1}, q_{1}}\left(Q^{a}\right)\right)$ and consequently 


$$
H^{P-p_{1}, q-q_{1}-1}\left(X, R^{p_{1}, q_{1}}\left(Q^{a}\right)\right) \text { and }{ }^{P} \mathcal{E}_{1, B}^{p-p_{1},(q-1)-\left(p-p_{1}\right)} \text { are zero. }
$$

It may well be that $R^{p_{1}, q_{1}}\left(Q^{a}\right)$ is isomorphic to the associated graded bundle $\operatorname{Gr} R^{p_{1}, q_{1}}\left(Q^{a}\right)$, as in the Grassmannian case discussed above. We did not investigate this question, since it is not necessary for our proof.

We have shown that under the condition $\left({ }^{*}\right)^{P} \mathcal{E}_{1, B}^{P, q-P}=H^{P, q}\left(X, \wedge_{r} E\right)$ is a subquotient of $H^{P, q}\left(Y, Q^{a}\right)$.

Now $Q^{a}$ is ample by

Lemma 6.2. Let $a=\left(a_{1}, a_{2}, \ldots\right)$ a strictly decreasing partition, and $s$ as above.

If $\mathcal{S}_{a_{s}} E$ is ample over $X$, then $Q^{a}$ is ample over $Y$.

Proof: See [D1], Lemmas 2.11 and 4.1.

To conclude the proof of Theorem 1.1, the group $H^{P, q}\left(Y, Q^{a}\right)$ vanishes under the condition of Kodaira-Akizuki-Nakano theorem but the condition $(*)$ implies this condition.

\subsection{Proof of Corollary 1.2.}

We need the following

Lemma 6.4. $\otimes_{i=1}^{l} S^{k_{i}} E \otimes_{j=1}^{m} \wedge^{s_{j}} E$ is ample $\Longleftrightarrow \mathcal{S}_{\lambda} E$ is ample, where $\tilde{\lambda}=(s_{1}, s_{2}, \ldots, s_{m}, \underbrace{1, \ldots, 1}_{k_{1} \text { times }}, \underbrace{1, \ldots, 1}_{k_{2} \text { times }}, \ldots, \underbrace{1, \ldots, 1}_{k_{m} \text { times }})$.

Proof:

$\mathcal{S}_{\lambda} E$ is direct summand of $\otimes_{i=1}^{l} S^{k_{i}} E \otimes_{j=1}^{m} \wedge^{s_{j}} E$.

Conversely for any $\mathcal{S}_{\lambda_{i}} E$ direct summand of $\otimes_{i=1}^{l} S^{k_{i}} E \otimes_{j=1}^{m} \wedge^{s_{j}} E$, we have $\lambda \succeq \lambda_{i}$, then we use Lemma 3.5.

Now

Among $\mathcal{S}_{\lambda_{i}} E$ where $\lambda_{i}=\left(r_{1}, r_{2}, \ldots\right)$, that appear as direct summands of $\otimes_{i=1}^{l} S^{k_{i}} E \otimes_{j=1}^{m} \wedge^{s_{j}} E$, the optimal condition $\sum_{i=1}^{m} r_{i}\left(d-r_{i}\right)$ is obtained by $\lambda$.

This concludes the proof of Corollary 1.2

\section{REFERENCES}

[KAN] Y. Akizuki, S. Nakano, Note on Kodaira-Spencer's proof of Lefschetz theorems, Proc.Jap.Acad. 30 (1954), 266-272.

[B] R.Bott, Homogeneous vector bundles, Ann. Math. 66 (1957), 203-248.

[D1] J.P. Demailly, Vanishing theorems for powers of an ample vector bundle, Invent. Math. 91 (1988), 203-220. 
[D2] B. Demazure, A very simple proof of Bott's theorem, Invent. Math. 33 (1976), 271-220.

[FH] W. Fulton, J. Harris Representation theory, Springer Verlag

[G] P.A. Griffiths, Hermitian differential geometry, Chern classes and positive vector bundles, Global analysis, paper in honor of Kodaira (1969), 185-251.

[KV] Kawamata, A generalization of Kodaira-Ramanujam's vanishing theorem, Math. Ann. 261 (1982), 43-46.

[L] F. Laytimi, On Degeneracy Loci, International Journal of Mathematics 6 (Vol 7) (1988), 203-220.

[LN] F. Laytimi, W. Nahm Vanishing theorems for product of exterior and symmetric powers, e-print math.AG/9809064.

[LP1] Le Potier, Cohomologie de la Grassmannienne à valeurs dans les puissances extérieures et symetriques du fibré universel, Math. Ann. 226 (1977), 257270.

[LP2] J. Le Potier, Annulation de la cohomologie à valeurs dans un fibré vectoriel holomorphe positif de rang quelconque, Math. Ann. 218 (1975), 35-53.

[M] I.G. Macdonald, Symmetric Functions and Hall polynomials, Clarendon Press, Oxford (1979) (1997), 401-416.

[M1] L. Manivel, Un théorème d'annulation pour les puissances extérieures d'un fibré ample, J. reine angew. Math. 422 (1991), 91-116.

[M2] L. Manivel, Vanishing theorems for ample vector bundles,, Invent.math.127 (1997), 401-416.

[S] A.J. Sommese, Submanifold of Abelian Varieties,, Math.Ann.233(1978), 229256 .

F. L.: Mathématiques - BÂt. M2, Université Lille 1, F-59655 VilLeneuve d'Asce Cedex, France

E-mail address: laytimi@agat.univ-lille1.fr

W. N.: Physikalisches Institut, Universität Bonn, Nussallee 12, D53115 BONN

E-mail address: werner@th.physik.uni-bonn.de 